Case Report

\title{
A Case Report of a Giant Adrenal Angiomyolipoma at Computed Tomography
}

Francesco Messina*, Lorena Turano and Nicola Arcadi

Unit of Radiology, Riuniti Hospital, Azienda Ospedaliera Grande Ospedale Metropolitano, Bianchi-Melacrino-Morelli, Italy

\section{Abstract}

Angiomyolipoma of the adrenal gland is an extremely uncommon tumor detected incidentally in studies for other pathologies. We present the case of a 55-yearold female patient with a giant incidental right adrenal angiomyolipoma, detected by Computed Tomography (CT), performed for abdominal pain.

Keywords: Adrenal angiomyolipoma; Computed tomography; MPR; Density

\section{Case Presentation}

A 55-year-old female patient presented at emergency department with abdominal pain since about a month, mostly in the left lower abdominal quadrants, and at a deep palpation [1,3]. No fever, and arterial oxygen saturation $\left(\mathrm{SaO}_{2}\right)$ was $96 \%$. The naso-pharyngeal sampling for SARS-CoV-2 was negative. Laboratory exams showed only a slight increase, a specific, of the values of C-reactive protein. It was decided firstly to perform an unenhanced CT, completed by a contrast-enhancement CT, which showed the presence of little diverticula of the descending colon. But CT also incidentally showed (Figure 1) the presence in the right adrenal gland of a giant, voluminous, mass occupying space (diameters of $10 \mathrm{~cm} \times 9 \mathrm{~cm}$ ), hypodense and with a mixed densitometric component (angio-myo and lipomatous), of which greater adipose/fatty like density values, almost all its surface, with blurred margins. Due to patient's symptoms and the results of complementary laboratoristic tests, the diagnosis was of a giant right adrenal angiomyolipoma. In the left adrenal gland CT had also shown the presence of another adipose like density mass (diameter $2 \mathrm{~cm}$ ), such as adrenal lipoma. The patient, after surgical consulence, is waiting for surgery to remove the voluminous right adrenal mass; meanwhile, she is carrying out an US and laboratory monitoring of it.

\section{Discussion}

Angiomyolipoma is an extremely uncommon tumor of the adrenal gland, detected incidentally. They are rare lesions, often arising in the kidney, and are part of a group of tumors with a diverse appearance known as tumors of perivascular epithelioid cell origin; extrarenal

Citation: Messina F, Turano L, Arcadi N. A Case Report of a Giant Adrenal Angiomyolipoma at Computed Tomography. Ann Clin Cases. 2021; 2(3): 1035.

Copyright: $\odot 2021$ Francesco Messina

Publisher Name: Medtext Publications LLC

Manuscript compiled: Jun $25^{\text {th }}, 2021$

*Corresponding author: Francesco Messina, Unit of Radiology, Riuniti Hospital, Azienda Ospedaliera Grande Ospedale Metropolitano, "Bianchi-Melacrino-Morelli", Via Giuseppe Melacrino n.21, 89124 Reggio Calabria, Italy, Tel: +39-3392641419; E-mail: fmessina1@ hotmail.it

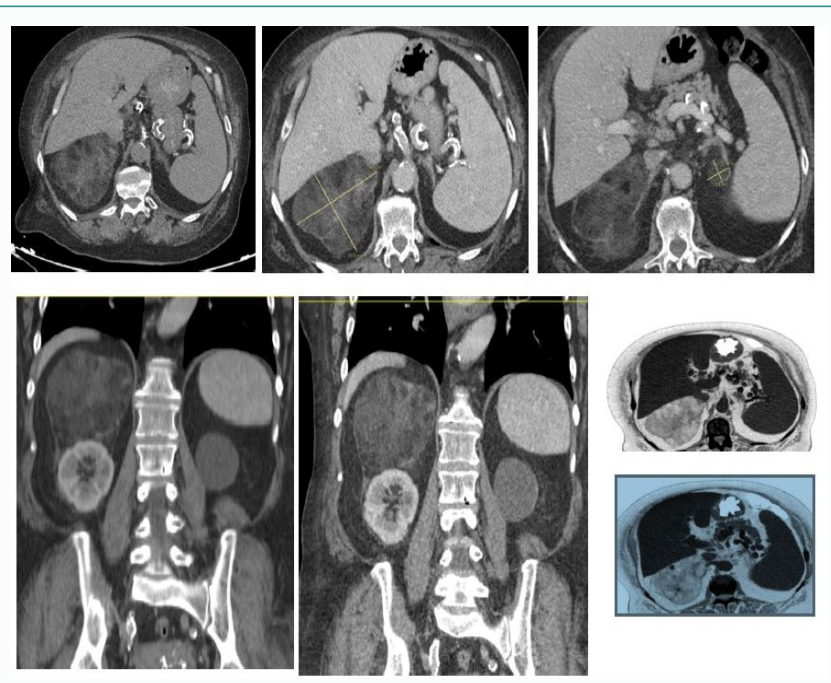

Figure 1: Contrast enhancement Computed Tomography reconstructions Axial MPR (upper line); coronal MPR (under line, on the left) and simil-MRI reconstructions (under line, on the right). CT had shown the presence of a giant, voluminous, right adrenal angiomyolipoma $(10 \mathrm{~cm} \times 9 \mathrm{~cm})$, and another one (left adrenal gland) of about $2 \mathrm{~cm}$. CT had well-defined the mixed densitometric components of the mass.

angiomyolipomas are extremely rare [4,5]. Angiomyolipomas represent the $0.7 \%$ of adrenal tumors and the $4 \%$ of adrenal lipomatous tumors; they are more frequent in male $(3: 2)$ with an average age of 54 years. Pathophysiologically, it is adrenal cortex cell metaplasia into reticuloendothelial cells, resulting from infection, chronic stress or adrenal gland degeneration. Histologically, the tumor is composed of mature fat tissue associated with normal haematopoietic tissue [6]. Most of the cases were observed in asymptomatic patients. If any symptoms occur, the most frequent would be low back pain. The studies showed that the adrenal incidentalomas, which include angiomyolipomas, are usually unilateral and located in the right gland. Its diagnosis is based on CT scan that identifies the fat percentage. Tumor size varies between $1 \mathrm{~cm}$ to giant lipomas (greater than 8 $\mathrm{cm}$ ) that reaching $20 \mathrm{~cm}$ and almost $3 \mathrm{~kg}$ of weight $[7,8]$. Surgical resection of lipoma is usually performed and is indicated when tumor is voluminous, compressive or at risk of hemorrhage. 


\section{Conclusion}

Adrenal angiomyolipomas are rare tumors, generally asymptomatic, that are usually incidentally found. The diagnosis is based, firstly, on Computed Tomography scan that identifies, especially on emergency, the fat percentage. So, CT had an important role in the diagnosis of adrenal angiomyolipomas, and also in the follow-up of the patients.

\section{Conflicts of Interest}

The authors certify that there is no conflict of interest with any financial organization regarding the material discussed in the manuscript.

\section{Patient Consent Statement}

The patient had confirmed her consense for the publication of our case report.

\section{References}

1. Lam Ak. Lipomatous tumours in adrenal gland: WHO updates and clinical implications. Endocr Relat Canc. 2017;24(3):R65-R79.
2. Lam KY, Chan AC, Ng IO. Giant adrenal lipoma: a report of two cases and review of literature. Scand J Urol Nephrol. 1997;31(1):89-90

3. Zhao J, Sun F, Jing X, Zhou W, Huang X, Wang H, et al. The diagnosis and treatment of primary adrenal lipomatous tumours in Chinese patients: 31 years follow-up study. Can Urologic assoc J. 2014;8(3-4):E132-6.

4. Alkhalifa AM, Aldossary MY, Abusultan AJ, AlQattan AS, Alsomali M, Alquraish F et al. Lipomatous tumors of adrenal gland: a case series of 5 patients and review of the literature. Int J Surg Case Rep. 2020;67:54-61

5. Godara R, Vashist MG, Singla SL, Garg P, Sen J, Mathur SK, et al. Adrenal angiomyolipoma: a rare entity. Indian J Urol. 2007;23(3):319-20.

6. D’Antonio A, Caleo A, Caleo O, De Dominicis G, Boscaino A. Monotypic epithelioid angiomyolipoma of the adrenal gland: an unusual site for a rare extrarenal tumor. Ann Diagn Pathol. 2009;13(5):347-50.

7. Lam Ky, Lo C. Adrenal Lipomatous tumour: a 30 years clinico-pathological experience at a single institution. J Clin Pathol. 2001;54(9):707-12.

8. Yener O, Ozelik A. Angiomyolipoma of the right adrenal gland. ISRN. Surg 2011:102743. 